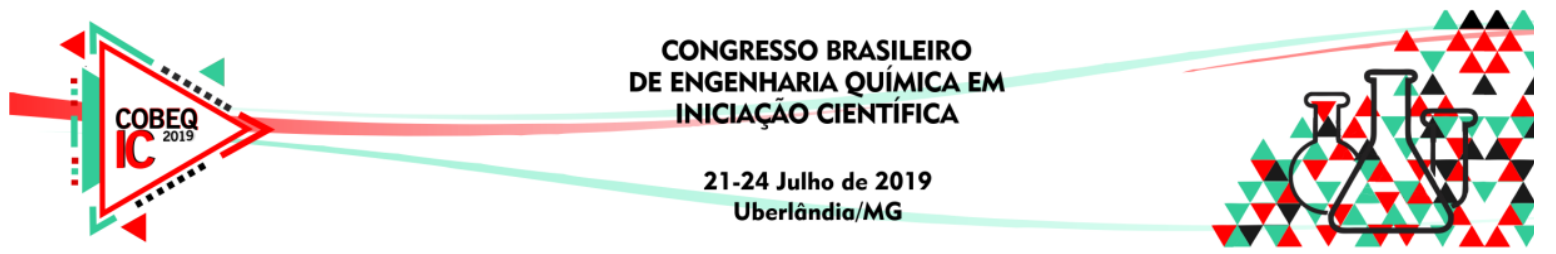

\title{
ESTUDO IN SILICO DO POTENCIAL DE APLICAÇÃO DOS ALCALOIDES ISOQUINOLÍNICOS DO GRUPO OCOTEA (LAURACEAE) NO DESENVOLVIMENTO DE FÁRMACOS
}

\author{
E. MOCHIUTTI ${ }^{1}$, A. L. S. CARVALHO ${ }^{1}$, R. L. C. SEHWARTZ ${ }^{1}$, D. S. B. BRASIL ${ }^{1}$ e M. C. \\ MARTELLI ${ }^{1}$ \\ ${ }^{1}$ Universidade Federal do Pará, Faculdade de Engenharia Química \\ E-mail para contato: mochiuttieric@gmail.com
}

\begin{abstract}
RESUMO - A constante procura por produtos que auxiliam no combate à patologias e/ou possuem propriedades terapêuticas movimenta a indústria farmacêutica. No presente trabalho, analisou-se o potencial de aplicação dos alcaloides Dicentrina, Isoboldina, Isocoridina e Ococriptina, pertencentes ao grupo Ocotea (Lauraceae), no desenvolvimento de fármacos através de um estudo in silico. Durante a pesquisa, utilizou-se de ferramentas online como os servidores PreADMET e PASS, além de softwares como MarvinSketch, Gaussian e Spartan para procedimentos como cálculos de otimização (utilizando-se o método DFT e o conjunto de bases B3LYP/6-31G*) e frequências. Para a análise dos resultados, comparou-se os mesmos com dados estatísticos presentes na literatura, além de ter sido aplicada também a regra dos cinco de Lipinski. Os resultados obtidos demonstraram que a Isoboldina é o composto mais estável e eletronegativo, enquanto a Dicentrina é o alcaloide mais eletropositivo. Além disso, as propriedades físico-químicas verificadas para cada molécula revelaram que todas podem ser candidatas a fármacos, com base na regra de Lipinski. O teste de AMES mostrou que a Ococriptina possui caráter não mutagênico, enquanto a Dicentrina apresentou resultados negativos durante os testes de carcinogenicidade em ratos. Ademais, todos os alcaloides devem apresentar uma excelente absorção intestinal, uma média permeabilidade nas células Caco-2 e elevadas chances de suas atividades biológicas serem ativas.
\end{abstract}

\section{INTRODUÇÃO}

Pode-se definir como alcaloides os compostos nitrogenados e farmacologicamente ativos, encontrados geralmente em angiospermas (Simões et al., 2004). Em relação a sua estrutura química, os alcaloides podem ser classificados como alcaloides verdadeiros, protoalcaloides e pseudo-alcaloides (Bruneton, 2001).

A família Lauraceae é uma das espécies botânicas mais interessantes para o reflorestamento de áreas degradadas, sua madeira é amplamente utilizada, assim como seus frutos (Lorenzi, 1992). Além disso, a presença de alcaloides isoquinolínicos no gênero Ocotea é algo a se destacar, onde compostos como a Dicentrina, Isoboldina, Isocoridina e Ococriptina foram isolados em trabalhos publicados na última década (Zanin e Lordello, 2007). 


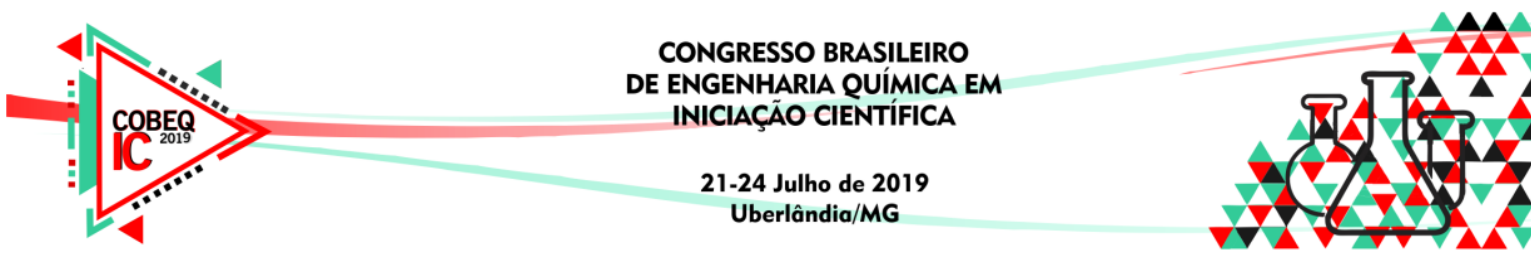

Segundo a Organização Mundial de Saúde, cerca de $65 \%$ a $80 \%$ da população mundial, em condições de pobreza e/ou sem acesso a medicina moderna, dependem essencialmente de plantas nos cuidados primários de saúde (Parker et al., 2007). No Brasil, o estudo relacionado a área de plantas medicinais se expandiu e se consolidou por volta do século XX, estando relacionado à implementação de instituições de pesquisa e à organização de disciplinas como botânica, química e farmacologia (Fernandes, 2014).

Com o avanço tecnológico, o emprego de técnicas computacionais no descobrimento e desenvolvimento de fármacos vem sendo bastante difundido atualmente. Segundo Kapetanovic (2008), as vantagens de se realizar estudos in silico incluem a economia de tempo e a otimização de resultados. Nesse contexto, o presente trabalho busca avaliar o potencial dos alcaloides Dicentrina, Isoboldina, Isocoridina e Ococriptina como fármacos.

\section{METODOLOGIA}

O estudo in silico dos alcaloides Dicentrina (ID: 1), Isoboldina (ID: 2), Isocoridina (ID: 3) e Ococriptina (ID: 4) foi desempenhado a partir dos modelos 2D de cada um, os quais foram desenhados no programa MarvinSketch, como mostra na Figura 1; cálculos de minimização de energia foram executados através do programa Gaussian; parâmetros como o método DFT e o conjunto de bases B3LYP/6-31G* foram utilizados. Além disso, cálculos de frequência foram executados com o intuito de confirmar os pontos estacionários, garantindo a inexistência de frequências imaginárias e mostrando que as estruturas correspondiam à uma conformação de energia mínima local (Souza et al., 2018).

Figura 1 - Estrutura bidimensional dos compostos analisados<smiles>COc1cc2c(cc1OC)-c1c3c(cc4c1C(CC2)N(C)CC4)OCO3</smiles>

Dicentrina<smiles>COc1cc2c(cc1OC)CC1c3c(OC)ccc(OC)c3-c3c(ccc(OC)c3O)C1C2</smiles>

Isocoridina<smiles>COc1ccc2c(c1O)-c1c(O)c(OC)cc3c1C(C2)N(C)CC3</smiles>

Isoboldina<smiles>COc1ccc2c(c1OC)-c1c(OC)c3c(c4c1C(CCC4C)C2)OCO3</smiles>

Ococriptina

Através do software Spartan, determinou-se as propriedades físico-químicas de cada composto, como energia do orbital molecular ocupado mais alto (HOMO), energia do orbital molecular desocupado mais baixo (LUMO), diferença entre HOMO e LUMO (GAP), massa 


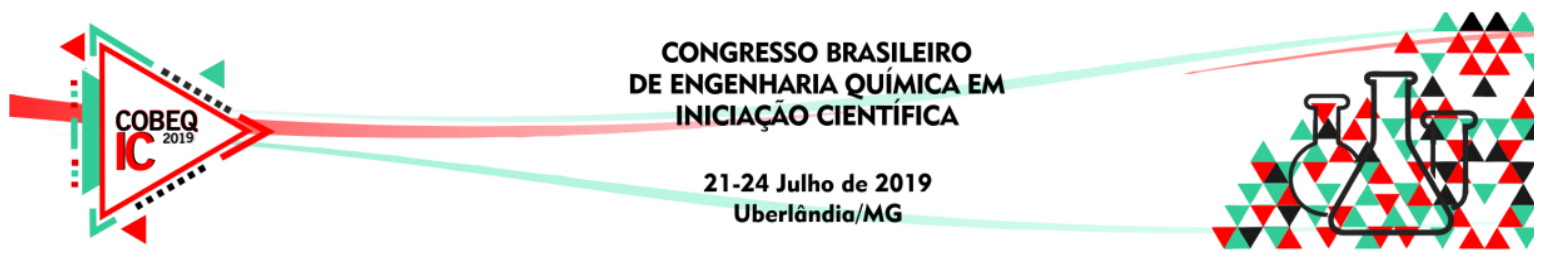

(MW), coeficiente de partição (Log P), doador de ligação de hidrogênio (HBD), aceitador de ligação de hidrogênio (HBA) e área de superfície polar (PSA). A partir de tais resultados, a regra dos cinco de Lipinski foi aplicada para verificar a viabilidade do uso dos compostos como fármacos (Lipinski et al., 1997).

As propriedades farmacocinéticas das moléculas foram obtidas através de servidores online. Informações referentes a absorção intestinal humana (HIA), permeabilidade celular in vitro (PCaco-2), mutagenicidade (teste de AMES) e carcinogenicidade (para ratos e camundongos) foram obtidas através do servidor PreADMET (Lee et al., 2003). Resultados referentes a possíveis atividades biológicas de cada composto foram obtidas através do servidor PASS (Poroikov et al., 2003)

\section{RESULTADOS E DISCUSSÕES}

A Tabela 1 apresenta as propriedades físico-químicas das moléculas, determinadas com o auxílio do programa Spartan.

Tabela 1 - Características físico-químicas dos compostos estudados.

\begin{tabular}{|c|c|c|c|c|c|c|c|c|}
\hline ID & E $_{\text {Hомо }}(\mathrm{eV})$ & E $_{\text {Lumo }}(\mathrm{eV})$ & $\mathrm{GAP}(\mathrm{eV})$ & $\mathrm{MW}(\mathrm{da})$ & HBD & HBA & Log P & PSA $\left(\AA^{2}\right)$ \\
\hline \hline 1 & $-5,24$ & $-0,59$ & 4,65 & 339,391 & 0 & 5 & $-2,82$ & 30,462 \\
\hline 2 & $-5,02$ & $-0,51$ & 4,51 & 327,38 & 2 & 5 & $-3,06$ & 45,057 \\
\hline 3 & $-5,11$ & $-0,58$ & 4,53 & 341,407 & 1 & 5 & $-2,95$ & 34,472 \\
\hline 4 & $-5,13$ & $-0,53$ & 4,6 & 355,39 & 1 & 6 & $-3,9$ & 45,001 \\
\hline
\end{tabular}

De acordo com Fleming (1976), quanto menor o valor para $\mathrm{E}_{\mathrm{HOMO}}$, mais eletronegativa será a molécula. Logo, a partir da análise dos valores descritos na Tabela 1, verifica-se que a Dicentrina é a molécula mais eletropositiva, pois apresenta o maior valor para a propriedade $\mathrm{E}_{\mathrm{HOMO}}(-5,24 \mathrm{eV})$, enquanto a Isoboldina é a mais eletronegativa por apresentar o menor valor para a mesma propriedade $(-5,02 \mathrm{eV})$.

De acordo com Santos et al. (2014), o valor do GAP é um importante indicador da estabilidade de uma molécula. Neste contexto, haja vista que a Isoboldina apresenta o menor valor de GAP em relação as demais moléculas $(4,51 \mathrm{eV})$, pode-se inferir que a mesma é o composto menos estável do grupo estudado, diferentemente da Dicentrina, a qual possui o maior valor de GAP $(4,65 \mathrm{eV})$ e pode ser considerada a mais estável entre as demais.

A regra dos cinco de Lipinski estabelece que uma má absorção de um candidato a fármaco é mais provável quando uma molécula apresenta mais de cinco doadores de ligação de hidrogênio, massa maior que $500 \mathrm{da}$, um valor de Log P maior que 5 e mais de dez aceitadores de ligação de hidrogêno (Lipinski et al., 1997). Portanto, pode-se verificar que todos os compostos podem ser bons candidatos a fármacos. Quanto aos valores verificados para a área de superfície polar (PSA), Pajouhesh e Lenz (2005) afirmam que compostos com PSA abaixo de $60 \AA^{2}$ são completamente absorvidos pelas células Caco-2 intestinais. Em relação as propriedades obtidas através do servidor PreADMET, as mesmas estão descritas nas Tabelas 2 e 3 . 
Tabela 2 - Propriedades ADME dos compostos estudados

\begin{tabular}{|c|c|c|}
\hline ID & Pcaco-2 $(\mathrm{nm} / \mathrm{s})$ & HIA (\%) \\
\hline \hline 1 & 56,519 & 97,758 \\
\hline 2 & 18,054 & 93,264 \\
\hline 3 & 45,373 & 95,657 \\
\hline 4 & 42,789 & 95,830 \\
\hline
\end{tabular}

Tabela 3 - Informações sobre mutagenicidade e carcinogenicidade dos compostos

\begin{tabular}{|c|c|c|c|}
\hline ID & Mutagenicidade & Carcinogenicidade (Ratos) & Carcinogenicidade (Camundongos) \\
\hline \hline 1 & Mutagênico & Não & Sim \\
\hline 2 & Mutagênico & Sim & Sim \\
\hline 3 & Mutagênico & Sim & Sim \\
\hline 4 & Não mutagênico & Sim & \\
\hline
\end{tabular}

Segundo Pérez et al. (2004), candidatos a fármacos que apresentam valores para a propriedade HIA maior ou igual a 80\%, apresentarão uma excelente absorção intestinal. Logo, pode-se constatar a partir da Tabela 2, que todas as moléculas se enquadram em tal classificação. Em relação a permeabilidade dos compostos nas células Caco-2, a classificação feita por Yazdanian et al. (1998) permite inferir que todos os compostos apresentam uma média permeabilidade, haja vista que todos os resultados para PCaco-2 estão no intervalo entre $4 \mathrm{~nm} / \mathrm{s}$ e $70 \mathrm{~nm} / \mathrm{s}$. Quanto ao teste de carcinogenicidade, Cunha et al. (2015) e Moreira et al. (2002) explicam que o teste de AMES é um método aplicado com o intuito de analisar a ação mutagênica de um composto em função de sua concentração, utilizando-se ratos e/ou camundongos. Nesse sentido, nota-se que a Ococriptina apresentou caráter não mutagênico e a Dicentrina apresentou resultados negativos durante os testes de carcinogenicidade em ratos. Ademais, os resultados obtidos através do servidor PASS estão dispostos na Tabela 5, onde pode-se observar as possíveis atividades biológicas dos compostos estudados.

Tabela 1 - Atividades biológicas possíveis dos compostos analisados

\begin{tabular}{|c|c|c|c|}
\hline Composto & Atividade & PA & PI \\
\hline \multirow{6}{*}{ Dicentrina } & Antitussive & 0,881 & 0,002 \\
\hline & Sigma receptor agonist & 0,88 & 0,002 \\
\hline & Respiratory analeptic & 0,84 & 0,006 \\
\hline & MAP kinase stimulant & 0,808 & 0,003 \\
\hline & 5 Hydroxytryptamine release stimulant & 0,762 & 0,018 \\
\hline & Antiparkinsonian. rigidity relieving & 0,758 & 0,003 \\
\hline \multirow{2}{*}{ Isoboldina } & Membrane permeability inhibitor & 0,773 & 0,015 \\
\hline & Neurotransmitter antagonist & 0,759 & 0,003 \\
\hline \multirow{6}{*}{ Isocoridina } & 5 Hydroxytryptamine release stimulant & 0,951 & 0,003 \\
\hline & UGT1A9 substrate & 0,814 & 0,004 \\
\hline & MAP kinase stimulant & 0,796 & 0,003 \\
\hline & Histamine release stimulant & 0,794 & 0,003 \\
\hline & Oxygen scavenger & 0,757 & 0,004 \\
\hline & Antineurotic & 0,753 & 0,026 \\
\hline \multirow{3}{*}{ Ococriptina } & Antitussive & 0,903 & 0,002 \\
\hline & Neurotransmitter uptake inhibitor & 0,881 & 0,002 \\
\hline & 5 Hydroxytryptamine release stimulant & 0,792 & 0,015 \\
\hline
\end{tabular}




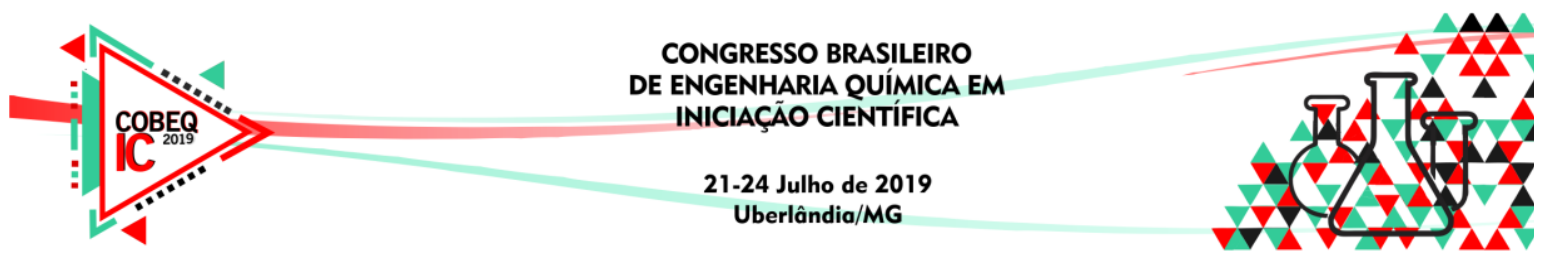

Segundo Lagunin et al. (2000), ao apresentar PA > 0,7, um composto possui uma elevada probabilidade ser ativo, embora suas chances de ser semelhantes a um fármaco já conhecido seja diretamente proporcional. Portanto, pode-se constatar que todos apresentam atividades biológicas com grandes chances de serem ativas, onde se destaca o efeito da Isocoridina como estimulante na liberação do neurotransmissor 5-Hydroxytryptamine (serotonina).

\section{CONCLUSÃO}

Logo, a partir deste trabalho pôde-se concluir a respeito do potencial de aplicação dos compostos estudados como fármacos. A regra de Lipinski permitiu inferir que todas as moléculas analisadas podem ser candidatas a fármacos, enquanto que as propriedades físicoquímicas, farmacocinéticas e de toxicidade apresentadas ao longo deste estudo demonstram que todas as moléculas apresentam o mesmo padrão de comportamento durante o processo de absorção intestinal e de permeabilidade nas células Caco-2.

Durante o estudo in silico, constatou-se que a dicentrina é o composto que mais se destaca em relação aos demais, haja vista que sua estabilidade e seus resultados no teste de AMES permitem concluir que a mesma é a molécula com maior potencial de aplicação como fármaco. Nesse sentido, pode-se direcionar a mesma para maiores testes experimentais, com o intuito de verificar sob quais condições ela pode ser utilizada pela sociedade.

\section{REFERÊNCIAS}

BRUNETON, J. Farmacognosia. Fitoquímica. Plantas Medicinales. $2^{\mathrm{a}}$ ed. Zaragosa: Editora Acribia, 2001.

CUNHA, E. L. et al. Computational investigation of antifungal compounds using molecular modeling and prediction of ADME/tox properties. Journal of Computational and Theoretical Nanoscience, v. 12, n. 10, p. 3682-3691, 1 out. 2015.

FERNANDES, T. M. Plantas medicinais: memória da ciência no Brasil. Editora FIOCRUZ, 2014.

FLEMING, I. Frontier Orbitals and Organic Chemical Reactions. $1^{\mathrm{a}}$ ed. New York: Wiley, 1976. $256 \mathrm{p}$.

KAPETANOVIC, I. M. Computer-aided drug discovery and development (CADDD): In silicochemico-biological approach. Chemico-Biological Interactions, v. 171, n. 2, p. 165-176, jan. 2008.

LAGUNIN, A. et al. PASS: prediction of activity spectra for biologically active substances. Bioinformatics, v. 16, n. 8, p. 747-748, 1 ago. 2000.

LEE, S. K. et al. The PreADME Approach: Web-based program for rapid prediction of physicochemical, drug absorption and drug-like properties. EuroQSAR 2002 Designing Drugs and Crop Protectants: Processes, Problems and Solutions. v. 2003. p. 418-420, 2003. 


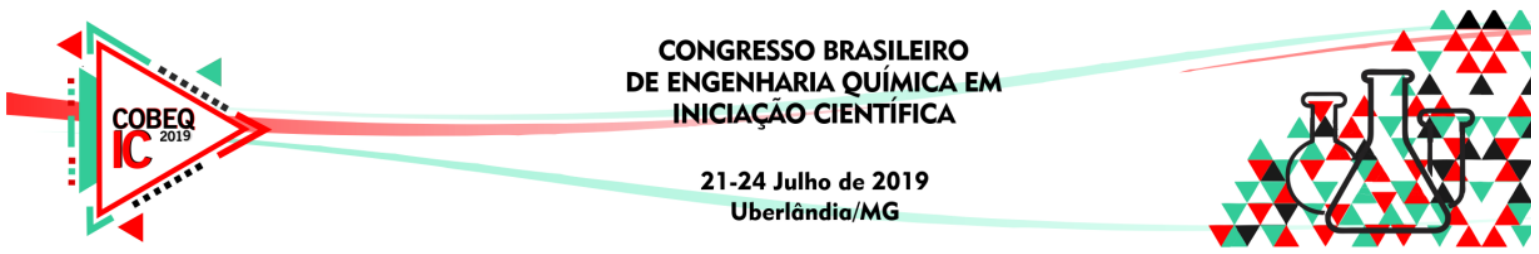

LIPINSKI, C. A. et al. Experimental and computational approaches to estimate solubility and permeability in drug discovery and development settings. Advanced Drug Delivery Reviews, v. 23, n. 1-3, p. 3-25, jan. 1997.

LORENZI, H. Árvores brasileiras: manual de identificação e cultivo de plantas arbóreas nativas do Brasil. Nova Odessa: Plantarum, 1992. $352 \mathrm{p}$

MOREIRA, R. R. D. et al. Avaliação da atividade mutagênica do extrato etanólico bruto de Paepalanthus latipes (Eriocaulaceae) e dos compostos flavonoídicos 7-metoxilados relacionados. Revista Brasileira de Farmacognosia, v. 12, n. 1, p. 11-19, 2002.

PAJOUHESH, H.; LENZ, G. R. Medicinal chemical properties of successful central nervous system drugs. NeuroRx, v. 2, n. 4, p. 541-553, out. 2005.

PARKER, A. G. et al. Antinociceptive effects of the aqueous extract of Brugmansia suaveolens flowers in Mice. Biological Research for Nursing, v. 8, n. 3, p. 234-239, 2007.

PÉREZ, M. A. C. et al. A topological sub-structural approach for predicting human intestinal absorption of drugs. European Journal of Medicinal Chemistry, v. 39, n. 11, p. 905-916, nov. 2004.

POROIKOV, V. V. et al. PASS Biological Activity Spectrum Predictions in the Enhanced Open NCI Database Browser. Journal of Chemical Information and Computer Sciences, v. 43, n. 1 , p. 228-236, 13 jan. 2003.

SANTOS, C. B. R. et al. Molecular Modeling: Origin, Fundamental Concepts and Applications Using Structure-Activity Relationship and Quantitative Structure-Activity Relationship. Reviews in Theoretical Science, v. 2, n. 2, p. 91-115, 1 jun. 2014.

SIMÕES, C. M. O. et al. Farmacognosia: da planta ao medicamento. $4^{\mathrm{a}}$ ed. Porto Alegre/Florianópolis: Editora UFRS/ UFSC, 2004.

SOUZA, T. N. V. et al. Adsorption of basic dyes onto activated carbon: Experimental and theoretical investigation of chemical reactivity of basic dyes using DFT-based descriptors. Applied Surface Science, v. 448, p. 662-670, ago. 2018.

YAZDANIAN, M. et al. Correlating partitioning and Caco-2 cell permeability of structurally diverse small molecular weight compounds. Pharmaceutical Research, v. 15, n. 9, p. 1490-1494, 1998.

ZANIN, S. M. W.; LORDELlO, A. L. L. Alcalóides aporfinóides do gênero Ocotea (Lauraceae). Química Nova, v. 30, n. 1, p. 92-98, fev. 2007. 